\title{
Next Generation Sequencing Based Comprehensive Molecular Analysis in Iranian Patients with Restless Legs Syndrome (RLS)
}

\section{ZAHRA ALIBABAEI}

Islamic Azad University Central Tehran Branch https://orcid.org/0000-0001-5778-5839

Ardeshir Hesampour ( $\nabla$ a.hesampour@gmail.com )

Central Tehran Branch,Islamic Azad University

Seyed.Mohammad.Mehdi Hamdi

Islamic Azad University Central Tehran Branch

Mohsen Najafi

Islamic Azad University Sari Branch

Hadiseh mohammadpour

Islamic Azad University Tehran North Branch

Rasoul Alizadeh

Iran University of Medical Sciences

Batoul Alibabaei

Islamic Azad University Kerman Branch

Research

Keywords: RLS syndrome, NGS, Molecoular analysis, Iranian Family, TANC1, ATXN7, MEIS1

Posted Date: June 8th, 2021

DOl: https://doi.org/10.21203/rs.3.rs-451536/v1

License: (c) (i) This work is licensed under a Creative Commons Attribution 4.0 International License.

Read Full License 


\section{Abstract}

Restless legs syndrome (RLS) is a common sensory-motor neurodegenerative disorder. It usually occurs at night and every 10 to 60 seconds with unpleasant sensations in the legs, burning and itching. Need to move and feel Uncomfortable legs improve with movement.In $20 \%$ of patients, symptoms can also include the arm and other parts of the body, which often occur in a more severe form of the disease. But generally, according to research, this syndrome is a multifactorial syndrome. These factors are divided into genetic and environmental or medical factors.

In this study, this disorder was investigated genetically and molecularly and the genes affecting the disease in Iranian population were identified by NGS method.

Materials and Methods: In this study, NGS and SEQUENCING methods were used to investigate the relationship between genes that influence disease and patients.

Conclusion: In this study, we found that ATXN7, MEIS1 and TANC1 genes are effective in restless legs syndrome. In this study, In this study, the effect of TANC gene on the development of restless legs syndrome in Iranian families was confirmed.

Discussion: The results of molecular analysis of restless legs syndrome using NGS method led to the identification of new gene loci on ATXN7 and TANC1 genes in Iranian population. Also, gene loci on MEIS1 gene, previous studies on its involvement in restless legs syndrome Confirmed.

\section{Introduction}

Restless Legs Syndrome (RLS), is a common sensorimotor of sleep disorders. Restless Legs Syndrome, or RLS, is often referred to as periodic leg movements (PLM) with involuntary movements and muscle spasms in the lower limbs. PLM may occur in either waking state (PLMW) or during sleep (PLMS). Pathological incidence of PLMS during sleep is found in about $80 \%$ of patients with RLS. The prevalence of RLS in the general population is about 3-5\% and about 2-3\% of the general population suffers from observable clinical symptoms (which occur twice a week with moderate to severe severity). It has been observed that with increasing age, the prevalence of the disease has increased and is higher in women than men. Studies strongly support the role of genetics in the development of this disease. Pharmacology and brain imaging also confirm the involvement of iron and opioid dopaminergic pathways in the brain and spinal cord in the incidence and exacerbation of the disease. Dopamine is required for smooth and purposeful muscle activity and movements, and as a result, disruption of these pathways usually leads to unwanted movements. Because of this, people with Parkinson's syndrome, which disrupts the dopamine pathways, are more likely to develop Restless Legs Syndrome. The RLS phenotype depends on the age of onset of the disorder. RLS or Restless Legs Syndrome is a multifactorial disease or polymorphism in which, in addition to genetics, the influence of environmental factors such as iron deficiency or other diseases such as diabetes and kidney disease has been proven. This disorder causes restlessness and severe concussions, mainly in the legs, due to decreased dopamine secretion in the brain neurons, which 
is why it is known as Restless Legs Syndrome. However, the symptoms of this disease also appear in different parts of the body, including the arms. This disease disrupts the sleep of the affected people at night ( figure 1).

The development of molecular diagnosis in the genetics of hereditary diseases is done to determine genetic defects along with the development of new technologies in this field. New technologies often lead to the rapid discovery of new genes and diseases that lead to molecular diagnosis, management, and disease care. Numerous studies have shown that $60 \%$ of patients have a positive family history. Phenocypes and possibly non-pantrans (non-pantrans) are a distinct genomic feature that does not occur in the individual's own phenotype and in contrast to pantrats. It sometimes happens that people carrying the same genes show different phenotypes. This problem, which depends on how the environment relates to and affects genes, is called "gene penetration" (PTR), making it difficult to identify a common separating haplotype in families.

Accurate identification of the candidate region is difficult due to non-familial, allelic and inorganic heterogeneous reasons. An important prerequisite for future successful genetic studies on Restless Legs Syndrome is the full availability of the phenotype of a large community of patients and their families. Studies also show that restless legs syndrome (RLS), autosomal dominant, is highly pervasive and is passed from parent to child. Numerous studies have been performed on the human genome to determine the genetic status of this disease and important results have been obtained. Despite numerous reports suggesting a genetic contribution to idiopathic RLS Ethiopia, numerous molecular genetic studies have attempted to identify genes that may be susceptible to the disorder. In particular, the encoded genes for GABA A receptor subunits, the glycine receptor alpha1 subunit gene, and the genes involved in dopaminergic transmission and metabolism have been analyzed and significant findings reported.

\section{Types of diseases}

Restless legs syndrome comes in two forms:

1. Primary type: Starting at an early age (less than 15 years) and with a set of common symptoms Genetics are associated in a family. Primary RLS (which accounts for $20 \%$ of all RLS cases) has an earlier onset, slower development, and a stronger family link, and is associated with dopaminergic (associated with dopamine transporters in the brain), iron deficiency, and sleep-wake cycles. Is.

2. Secondary type: usually starts at older ages and is related to environmental factors. Secondary causes of RLS include iron deficiency, kidney failure, peripheral neuropathy, diabetes, MS, Parkinson's disease, pregnancy, and drugs that stimulate RLS, such as neuroleptics, selective serotonin reuptake inhibitors, lithium (and neuropathy), neuropathy, radiculopathy Metabolic disorders such as iron deficiency, diabetes, $B$ vitamin deficiency, thyroid dysfunction (and rheumatic diseases) are rheumatoid arthritis.(Alibabaei, 2018) 


\section{Evaluation of people with genetically restless legs syndrome using NGS method to determine the genes involved in the development of the disease:}

Using the NGS method, or the study of exons, can help in the path of molecular diagnosis based on the disease panel. By examining the sequence of the exome or genome, a set of disease-causing genes can be reached. Exon examination, in addition to determining disease-causing mutations in index genes, can also be a good way to diagnose diseases and related new genes.

In this study, we examined the exon profile of infected individuals and examined the possibility of undetermined genetic mutations and new disease-related determinants in the Iranian population.

\section{The main hypotheses of the research}

\section{Family studies and possible genetic predictions}

According to genetic studies, restless legs syndrome is a disease of genetic origin and the role of genetics in the occurrence of this disease has been proven. In this study, blood samples were taken from 30 members of three Iranian families who were involved in severe to mild degrees of the disease, and by extracting DNA from the blood samples of the subjects, the possible genes involved in the development of the disease were examined.

\section{Inheritance pattern studies}

Although previous studies have shown that the disease follows an autosomal dominant pattern, but considering that this study was conducted for the first time in Iran, the study of hereditary patterns and how the disease is transmitted from parents to children was examined.

\section{Materials And Methods}

In order to conduct this study, 30 blood samples were collected at the rate of $5 \mathrm{cc}$ from three different genealogies from Fars, Tehran and Mazandaran provinces. A sample from each family was selected as a control and examined in the study. Patients with Restless Legs Syndrome were identified based on their symptoms and family history with the approval of a neurologist. Before sampling, patients were examined for non-involvement of non-hereditary factors such as iron deficiency, which is the most important non-genetic cause of the disease. Patients were justified in terms of the type of research and its stages, and special research questionnaires were completed with the help of the research community. Questionnaire questions were selected based on the research design. The questionnaire included questions from patients about symptoms, time and severity of symptoms, family history, medications, history of certain diseases such as diabetes, hemodialysis, Parkinson's and mental disorders. Also, patients with restless legs syndrome who were receiving drug treatment were identified and the type of drug used was determined. Age, sex, geographical location and family relationship of patients with each other were determined and genealogy was drawn. Based on the interpretation of the questionnaire and the study of previous research in other countries, the role of heredity in the disease was confirmed and 
blood samples were collected from patients and transferred to Tehran for further treatment. Prior to blood sampling, patients with restless legs syndrome in these families were identified through a questionnaire. Patients were evaluated for other environmental factors affecting the disease such as iron deficiency and diabetes and other environmental factors and according to the genealogy and ensuring the role of heredity in these patients, sampling was performed. Samples were placed in tubes for collecting human k3 blood samples containing EDETEA and marked for ease of use of samples 1 to 30 .

\section{Preparation of samples}

At this stage, the blood samples were taken out of the freezer at room temperature to return from freezing and return to normal. The specimens were placed vertically under the hood. In the laboratory, the study site was a laminar hood.

\section{Extraction of genomic DNA from blood}

After taking blood samples from individuals, DNA extraction from the samples began. At this stage, according to the protocol in the kit, first RBC was lysed in several stages and the samples were centrifuged and after complete lysis, the obtained sample was used for extraction.

\section{Mapping the genome and conducting studies on it to obtain a specific genetic link}

In this study, we have investigated the molecular causes of Restless Legs Syndrome using the NGS method using the experimental method. For this purpose, the DNA sample of a person with severe restless legs syndrome was selected and NGS was performed. Then, by analyzing the obtained data, 3 genes:TANC1,ATXN1 and MEIS1 were selected as the final genes and a primer was designed for these genes. Of these three genes, only the MEIS1 gene is a common gene known in Restless Legs Syndrome, and the other two genes were tested for the first time and new variants were identified.

The final product of PCR, after electrophoresis, samples with suitable bands were selected for sequencing or sequencing and sent for sequencing. Finally, sequence analysis was performed and new variants effective in the incidence of disease were identified.

\section{Study on candidate genes}

After selecting the final genes, primers were designed and PCR was applied to all samples and the PCR product was sent for sequencing. Then, by analyzing the obtained sequences, a study was performed on the candidate genes and the effect of these genes on the incidence of the disease.

\section{NGS}

Exom sequencing data, due to the high volume of information they need, requires special software to study and study. The primary data obtained from sequencing devices is often in FASTQ format, in which there are components of each sequence that is a hack of four types of openings, along with other information that indicates the reading quality of each base. FASTQ data are readings of a certain length, 
the number of which varies depending on the size of the cover (for example, with a coverage of 150 , about 100 million readings are obtained). The stages of analyzing exome data are generally divided into 5 stages: quality control and correction of readings, alignment, calling variants, screening (filtering), writing reports of each variant.

Exom data were analyzed using CLC genomics workbench and Biomedical genomic workbench software, which generally includes the following 6 steps:

A) Quality control and correction of readings

At this stage, the quality of each reading as well as each of the bases was evaluated. After determining the qualities, the correction was performed, so that if the game had a score of less than 20 , it was removed. A person's score is calculated according to the following equation:

$Q=1010 g p / 1-p$

At this stage, other information that showed the accuracy of the sequencer was checked, such as G-C content, reading length and number of repetitions. Adapters that were added to each of the readings in the sequencing step were also removed in this step.

B) Alignment

After the corrections made in the previous step, the readings that have passed that step are aligned with the reference human genome, which uses different algorithms.

Some examples of common algorithms for aligning exom data are:

1. Burroes-Wheeler Transfermation (BWT)

2. Smith-Waterman (SW)

3. Dynamic programming, Hash-based index

4. Seed-and-extend approach.

The human reference genome in this study was hg19 (Human reference genome).

C) Post-alignment processes

These processes included the following 2 steps:

1-Elimination of duplicates: When readings are attached to the reference genome to match it, there are too many connections in some parts of the genome, and this causes problems in later stages due to the high volume of these sequences, so with Given the quality and degree of similarity of duplicate sequences, some of them are omitted. 
2- Re-alignment: In the alignment stage, some bases are placed awkwardly and others are placed correctly. The mismatch indicates that our sequence is similar to the reference genome sequence, and that mismatch may indicate a SNP. However, in some cases, this is due to a poorly aligned process. Rearranging is an attempt to eliminate these inconsistencies and empty spaces created during alignment by using algorithms. The alignment performed at this stage is called the local alignment.

D) Calling variants

At this point, all the bases that are awkwardly aligned with the reference genome sequence are identified.

They turn. These base changes can take the form of single nucleotide polymorphisms, addition, deletion, variation in the number of replications, and structural variation.

E) Screening

In humans, there can be variations of the game, and this variation does not cause disease. At this stage, non-pathogenic variants can be isolated using common variants obtained from the genomic projects of different individuals. These projects include the Thousand Genomes Project, the EXAC Project, the NHLBI Exome Project, the SNP Database, the DiscoverEHR Project, as well as local projects involving people in a specific region (such as Iranum). Another concept in the screening phase is the minimum allelic frequency. This means that the frequency of the desired alleles that are studied in different projects, if it is less than a minimum value, that allele is considered rare. Screening is also performed at this stage, based on the type of inheritance pattern, the elimination of large recurrences, and the isolation of areas that are likely to be unrelated to the disease.

C) Write reports of each variant

The last step in analyzing Exome data is to write reports for each variant. At this stage, the number of variants has been greatly reduced due to their screening compared to the first case. Using different databases with information on different genes and alleles, we begin to examine the variants. These databases contain information on biological, molecular and cellular pathways and through them the relationship between the desired variant and the disease can be investigated. These databases include the SNP RSID database,

The DDD (Deciphering Developmental) study cited articles in various databases such as pubMed, the OMIM database, and COSMIC cancer information.

In addition to examining the above databases, predicting the pathogenicity of a variant is done by different algorithms. The most famous of them are SIFT, GWAVA, POLYPHEN and CADD. Each of these patterns scores the variants, which indicates the pathogenicity of that variant.

The WES test was performed with a 150X cover with the Illumina Hiseq 4000 system platform with a read length of 30 million and $8.5 \mathrm{~GB}$ of data was generated. 


\section{data analysis:}

In order to align the sequences, custom software such as NextGenMap was used to create SAM / BAM files.

Variant calling was performed using the GENOME ANALYSIS TOOLKIT (GATK) tool and databases including NCBI OMIM, HGMD and 1000 Genomes were used.

VCF files were annotated using ClinVar and EmVar.

PolyPhen and SIFT software were used to predict whether the displaced amino acid was likely to be detrimental to the function of the protein produced, based on the degree of retention of the displaced evolution during evolution.

The quality of the sequenced data was first checked by FastQC software version 0.11.3 (Andrews, 2010). Versatile software version 0.36 was used to filter the raw data credit and sort the low open quality data at both ends, and readings less than 36 were not considered.

Low-reading readings (Phred Score, $Q \geq 30$ ) were used for bioinformatics studies.

\section{SANGER SEQUENCING}

After analyzing the data and identifying the candidate genes, 30 samples were examined, sequenced and using websites ensembl,ncbi,mutation tester,varsome and finchtv,clc,dnstar and igv softwars,final results were extracted.

\section{Results}

This study was conducted on a community with Restless Legs Syndrome in Iran. According to previous research and proving the role of geographical environment in the occurrence of genetic mutations in infected people, samples were selected from three geographical areas of Fars, Tehran and Mazandaran provinces. All participants in the study completed the questionnaire according to the standards of the World Association of Restless Legs Syndrome. All patients had a strong family history or family history. No mutation or disease-related genetic disorder was observed in the control samples. The age of the patients was between 45-10 years and according to the research done from childhood, they had symptoms of the disease. Patients show varying degrees of disease from mild to severe. In the study population, in a 12-year-old girl, mild symptoms of the disease were observed, which in molecular studies also carries the identified variant. The results showed that $10 \%$ of patients were women and $6.6 \%$ were men. These data show that, as previously demonstrated, women are more likely to develop Restless Legs Syndrome than men. The role of heredity in the development of this syndrome was also proven in this study.

Based on the sequencing analysis of genes TANC1, the following results were obtained: 


\section{1-TANC1:}

The TANC1 gene was identified as the main cause of the disease, because this mutation was observed in all three generations F1, F2, F3. This mutation was observed in infected women.

chr2:159954270G $>A$ : The gene locus identified for this gene is located on the long arm of chromosome 2, region 2, band 4 and subband 2 and on exon 27.

The mutation is of the synonymous single nucleotide type. In this sequence, $\mathrm{G}$ is replaced by $\mathrm{A}$. Heterozygous and variant was identified as a pathogen.

This variant was common in 5 patients from 2 pedigree. four patients were of the one pedigree and mutations were observed in three generations of F1, F2 and F3.

Pedigree of a family with Restless Legs Syndrome with mutations in the TANC1 gene

\section{2-ATXN7}

Based on the results of this study, 8 variants were observed in the study population. the following variant was identified as a pathogen in three patients. variant chr3:63898457:G>A was observed in three patients with the same pedigree. Although due to the abundance of this variant in a pedigree, it can be introduced as a pathogen, but more research is needed. All three carriers of this variant had mild to severe restless legs syndrome. One patient was male and the other two were female.

\section{3-MEIS1}

This gene is known to be the main cause of Restless Legs Syndrome. In our study population, this woman showed the highest rate of mutation in the Iranian family. This confirms previous studies on the pathogenicity of this gene. In this study, 12 variants were identified for the MEIS1 gene. This site is located in the protein encoding region.

chr2:66664990T>G :According to studies, this mutation is one of the very rare mutations in the copper gene that before this study, only one case was identified in North Asia, and this is the second case of this mutation in the Iranian population studied in two The genealogy was observed. This mutation is on the $2 p 14$ locus of chromosome 3.The effectiveness of this variant in the development of restless legs syndrome requires further studies and research.

\section{Discussion}

Based on the results of this study, the effect of all three genes on the occurrence of restless legs syndrome in the studied families was confirmed. The TANC1 gene was identified as the main cause of the disease, because this mutation was observed in all three generations F1, F2, F3, and most importantly, this mutation is present in infected people in all three generations. 
This study shows that mutations in the TANC1 gene are mainly seen in women with restless syndrome. While known mutations in the ATXN7 gene are more common in infected men. But in the case of the MEIS1 gene, no significant gender differences are observed, and most mutations occur almost equally in men and women. The highlight of this study was the observation of a variant in the TANC1 gene that the female patient, who inherited the disease from a homozygous father, had a pure homozygous mutation. The table below shows the frequency of genes in people with mutations.(table1)

According to the evidence, this disease is autosomal dominant type with high penetration power. The important point is that This variant was observed in both homozygous and heterozygous forms. The frequency of variants is higher in women than men.

This confirms previous observations that restless legs syndrome is more common in women than men. This mutation was observed in the age group of 30-50 years. However, the identification of an infected 14-year-old girl needs further investigation.(table 2)

In this study, the disease was observed autosomally in the Iranian population. Examination of the data confirms the role of polymorphism in this disease.

According to the results of this study, it can be said that Restless Legs Syndrome is an autosomal dominant disease in the Iranian population and the discovery of new gene loci in the Iranian population proves the effect of environmental and geographical conditions on the incidence of the disease. This has already been confirmed in research on different families of other races in other countries. This study showed that restless legs syndrome has a genetic origin in the studied patients and confirms the previous data about restless legs syndrome.(table3)

\section{Conclusion}

The aim of this study was to investigate the molecular restlessness syndrome in Iranian society. This study was performed on families from Fars, Tehran and Mazandaran provinces. Sequence analysis showed that in the Iranian population studied, all three genes obtained from the patient's NGS analysis had an effect on the incidence of the disease(table4).

TANC1 has been confirmed as a new gene. On this gene, 1 variant was identified at a locus on the long arm of chromosome 3 , in four samples that were common in patients with close kinship (sister, brother, father and daughter). This position was higher in female patients, which confirms previous data on the prevalence of this disease in women.

ATXN7 In this gene, 8 variants were identified on the short arm of chromosome 2, in the Iranian family.

MEIS1 This gene has long been studied and confirmed as an effective candidate gene for restless legs syndrome worldwide. The presence of gene variants in this gene in the Iranian family also confirms the role of MEIS1 in the development of restless legs syndrome in the Iranian population. 11 variants were identified on this gene. 
It is hoped that in the future, with further studies and studies on more patients with restless legs syndrome, these new findings can be further examined and confirmed. Also, the study of the protein structure of pathogenic mutations can help how these mutations work. Slowly It is hoped that in the near future, genetic diagnosis kits will be designed using mutations identified in the Iranian population. These kits will play an effective role in faster identification of patients.

Gene therapy, which has gained its place in genetics today as a new and effective method in the treatment of genetic diseases, could be the next step in further studies.

\section{Declarations}

\section{Acknowledgements}

This study was conducted during my postgraduate studies in the Department of Biology, Faculty of Basic Sciences, Azad University, Tehran Branch. The authors would like to thank the Faculty of Basic Sciences, Director of the Department of Biology, for approving this study. The authors would like to thank Dr. Javad Setareh, a psychiatrist and faculty member of Mazandaran University of Medical Sciences, for their excellent cooperation and support. Dr. Jahantab's laboratory and Ms. Saba Malekian's laboratory and all members of the analysis team and the computer unit are also worthy of special mention for their contribution.

\section{References}

1. A case of restless leg syndrome in a family with LRRK2 gene mutation.. De Rosa A, Guacci $A$, Peluso S, Del Gaudio L, Massarelli M, Barbato S, Criscuolo C, De Michele G. Int J Neurosci. 2013 Apr;123

2. A direct interaction between two Restless Legs Syndrome predisposing genes: MEIS1 and SKOR1, Helene Catoire, Faezeh Sarayloo, Karim Mourabit Amari, Sergio Apuzzo, Alanna Grant, Daniel Rochefort, Lan Xiong, Jacques Montplaisir, Christopher J. Earley, Gustavo Turecki, Patrick A. Dion \& Guy A. Rouleau, (2018)

3. A Domestic Diagnosis System for Early Restless Legs Syndrome Based on Deep Learning Zhou P1, Luojie Huang,Huang L1, Zhao Q1, Xiao W1, Li S1, Chinese Journal of Medical Instrumentation, 28 Feb 2019,Exome Sequencing in a Family With Restless Legs Syndrome- 1Section of Clinical and Molecular Neurogenetics at the Department of Neurology, University of Lu“ beck, Lu“ beck, Germany; 2Department of Psychiatry and Psychotherapy, University of Lu“ beck, Lu“ beck, Germany; 3Center for Biomedicine; European Academy Bozen/ Bolzano, Bolzano; Italy; Affiliated Institute of the University of Lu“ beck, Lu“ beck, Germany; 4Department of Neuropediatrics, University of Kiel, Kiel, Germany; 5Behavioral Neurology and Movement Disorders Unit, Department of Neurology, Istanbul Faculty of Medicine, Istanbul University, Istanbul, Turkey; 6Department of Human Genetics,2012 University of Lu“ beck, Lu“ beck, Germany.2012

4. A Genetic Risk Factor for Periodic Limb Movements in Sleep, Hreinn Stefansson, Ph.D., David B. Rye, M.D., Ph.D., Andrew Hicks, Ph.D., Hjorvar Petursson, B.Sc., Andres Ingason, B.Sc., Thorgeir E. 
Thorgeirsson, Ph.D., Stefan Palsson, M.S., Thordur Sigmundsson, M.D., Albert P. Sigurdsson, M.D., Ingibjorg Eiriksdottir, B.Sc., Emilia Soebech, B.Sc., Donald Bliwise, Ph.D, (2207)

5. A review of sleep research in patients with spinal cord injury ria Dreier Thøfner Hultén,Fin BieringSørensen,Niklas Rye Jørgensen ORCID Icon \&Poul Jørgen Jennum,Published online: 04 Dec 2018

6. A TRAPPC6B splicing variant associates to restless legs syndrome* Paolo Aridon a, b, 1, Maurizio De Fusco a, 1, Juliane W. Winkelmann c, d, e, Marco Zucconi f,Valentina Arnao b, Luigi Ferini-Strambi f, Giorgio Casari. (2016)

7. Acceptability and feasibility of a 12-week yoga vs. educational film program for the management of restless legs syndrome (RLS): study protocol for a randomized controlled trial

8. An evaluation of sleep quality and the prevalence of restless leg syndrome in vitamin D deficiency Tuncay Çakır, Gülsüm Doğan, Volkan Subaşı, Meral Bilgilisoy Filiz, Nur Ülker, Şebnem Koldaş Doğan \& Naciye Füsun Toraman Acta Neurologica Belgica volume 115, pages623-627(2015)

9. Animal models of RLS phenotypes,Richard allen,Nathan Donelson,2016

10. Antoni Cortés,Estefanía Moreno,Mar Rodríguez-Ruiz,Enric I. Canela \&Vicent Casadó,2016.

11. Association between restless legs syndrome and other movement disorders View ORCID ProfileHortensia Alonso-Navarro, Elena García-Martín, José A.G. Agúndez, View ORCID ProfileFélix Javier Jiménez-Jiménez First published April 19, 2019,

12. Association Between the rs1229984 Polymorphism in the Alcohol Dehydrogenase 1B Gene and Risk for Restless Legs Syndrome, Félix Javier Jiménez-Jiménez, MD, PhD, Javier Gómez-Tabales, BsC, Hortensia Alonso-Navarro, MD, PhD, Martín Zurdo, MD, Laura Turpín-Fenoll, MD, PhD, Jorge MillánPascual, MD, Teresa Adeva-Bartolomé, MD, PhD, Esther Cubo, MD, PhD, Francisco Navacerrada, MD, Ana Rojo-Sebastián, MD, Sleep, Volume 40, Issue 12, December 2017

13. Association Between Vitamin D Receptor rs731236 (Taq1) Polymorphism and Risk for Restless Legs Syndrome in the Spanish Caucasian Population Félix Javier Jiménez-Jiménez, MD, PhD, Elena García-Martín, MD, PhD, Hortensia Alonso-Navarro, MD, PhD, Carmen Martínez, MD, PhD, Martín Zurdo, MD, Laura Turpín-Fenoll, MD, PhD, Jorge Millán-Pascual, MD, Teresa Adeva-Bartolomé, MD, PhD, Esther Cubo, MD, PhD, Francisco Navacerrada, MD, Ana Rojo-Sebastián, MD, Lluisa Rubio, MD, Sara Ortega-Cubero, MD, Pau Pastor, MD, PhD, Marisol Calleja, CN, José Francisco Plaza-Nieto, Belén Pilo-De-La-Fuente, MD, PhD, Margarita Arroyo-Solera, MD, Esteban García-Albea, MD, PhD, and José A.G. Agúndez, MD, PhD. 2015 Nov; 94(47): e2125.

14. Aurélien Hacquard, Laurence Hugueny, Jeffrey Hubbard, Ulker Kilic-Huck,Valérie Wolff, Patrice Bourgin,2016

15. Author links open overlay panelL.Lebrato HernándezM.Prieto LeónN.A.Cerdá FuentesA.J.Uclés SánchezJ.L.Casado ChocánM.Díaz Sánchez.2019

16. Autonomic dysfunction in restless legs syndrome, Yuksel ErdalEmail authorOzlem AkdoganMecbure NalbantogluGokce KavasogluUfuk Emre, 14 September 2019

17. Autosomal dominant restless legs syndrome maps on chromosome 14q, Maria Teresa Bonati, Luigi Ferini-Strambi, Paolo Aridon, Alessandro Oldani, Marco Zucconi, Giorgio Casari,2003 
18. Autosomal Dominant Restless Legs Syndrome Maps to Chromosome 20p13 (RLS-5) in a Dutch Kindred Antonetta M.G. Sas, MD,1 Alessio Di Fonzo, MD, PhD,2,3 Stef L.M. Bakker, MD, PhD,4 Erik J. Simons,2 Ben A. Oostra, PhD,2 Anneke J. Maat-Kievit, MD, PhD, Movement Disorders Vol. 25, No. 11, 2010, pp. 1715-1722

19. Benzodiazepines for restless legs syndrome,KARLA CARLOS,CAMLIA DM,LUCIA RADO,MARCH2017

20. Brain imaging and networks in restless legs syndrome, Author: Giovanni Rizzo, Xu Li, Sebastiano Galantucci, Massimo Filippi, Yong Won Cho ,2016

21. Central and peripheral nervous system excitability in restless legs syndrome Giuseppe Lanza $\mathrm{a}^{*}$, Cornelius G. Bachmann b, Imad Ghorayeb c,d, Yuping Wang e, Raffale. Ferri a, Walter Paulus fCentral and peripheral nervous system excitability in restless legs syndrome, Sleep Medicine (2016),

22. Childhood-onset Restless Legs Syndrome: Clinical and Genetic Features of 22 Families Hiltrud Muhle, MD,1* Anja Neumann, BS,1,2 Katja Lohmann-Hedrich, PhD,2 Thora Lohnau, BS,2 Yang Lu, MD,1,2,5 Susen Winkler, BS,2 Stephan Waltz, MD,3 Anke Fischenbeck, MD,1 Patricia L. Kramer, PhD,4 Christine Klein, MD,2 and Ulrich Stephani, MD1. Movement Disorders Vol. 23, No. 8, 2008, pp. 111311212008

23. Clinical, polysomnographic, and genetic characteristics of restless legs syndrome: A study of 133 patients diagnosed with new standard criteria, Dr. Jacques Montplaisir Sylvie Boucher Gaétan Poirier Gilles Lavigne Odile Lapierre Paul Lespérance,1997

24. Co-occurrence of Restless Legs Syndrome and Parkin Mutations in Two FamiliesSusanna Adel, MD,1,2 Ana Djarmati, MS,1,3 Kemal Kabakci, MD,1,3 Irene Pichler, BSc,4 Cordula Eskelson, MD,1,3 Thora Lohnau, BS, 1,3 Norman Kock, MD,1,3 Johann Hagenah, MD,1 Katja Hedrich, PhD,1,3 Eberhard Schwinger, MD,3 Patricia L. Kramer, PhD,5 Peter P. Pramstaller, MD,1,2,4 and Christine Klein, MD1,3*.2005

25. Decreased serum ferritin may be associated with increased restless legs syndrome in Parkinson's disease (PD): a meta-analysis for the diagnosis of RLS in PD patients. Kelu Li,Bin Liu,Fang Wang,Jianjian Bao,Chongmin Wu,Xiaodong2019

26. Department of Neurology, Govind Ballabh Pant Postgraduate Institute of Medical Education and Research, Academic Block, Room No 507, New Delhi - 110 009.India. Panda AK, Pandey S. Identifying risk factors for restless leg syndrome. Neurol India 2019;67:660-1

27. Depletion of Limiting rDNA Structural Complexes Triggers Chromosomal Instability and Replicative Aging of Saccharomyces cerevisiae, Ryan D. Fine, Nazif Maqani, Mingguang Li, Elizabeth Franck and View ORCID ProfileJeffrey S. Smith, May 1, 2019

28. Determinants of Nocturnal Cardiovascular Variability and Heart Rate Arousal Response in Restless Legs Syndrome (RLS)/Periodic Limb Movements (PLMS)

29. Differential Dopamine D1 and D3 Receptor Modulation and Expression in the Spinal Cord of Two Mouse Models of Restless Legs Syndrome Samantha Meneely1, Mai-Lynne Dinkins1, Miki Kassai1, Shangru Lyu2, Yuning Liu2, Chien-Te Lin1,3, Kori Brewer4, Yuqing Li2,5 and Stefan Clemens1*. 
30. Dopaminergic Agents in Rheumatoid Arthritis Silvia Capellino,Journal of Neuroimmune Pharmacology (2019)

31. Dopaminergic treatment of restless legs syndrome in spinal cord injury patients with neuropathic pain. Hatice Kumru, Sergiu Albu, Joan Vidal, Manuela Barrio \& Joan Santamaria Spinal Cord Series and Cases volume 2, Article number: 16022 (2016)

32. Dual roles of mitochondrial fusion gene FZO1 in yeast age asymmetry and in longevity mediated by a novel ATG32-dependent retrograde response, James C. JiangStefan W. StumpferlS. Michal Jazwinski, February 2019

33. Effective treatment of Restless Legs Syndrome by Safinamide in Parkinson's Disease patients. Claudio Liguori, Alessandro Stefani, Nicola Biagio Mercuri, Mariangela Pierantozzi UOC Neurologia e Neurofisiopatologia, Dipartimento di Medicina dei Sistemi, Università degli Studi di Roma,2017

34. Evidence for further genetic locus heterogeneity and confirmation of RLS-1 in restless legs syndrome, Juliane Winkelmann MD Peter Lichtner PhD Benno Pütz PhD Claudia Trenkwalder MD Stephanie Hauk MD Thomas Meitinger MD Tim Strom MD Bertram Muller-Myhsok PhD, 25 August 2005

35. Exome Sequencing in a Family With Restless Legs Syndrome. Anne Weissbach, MD,1 Katharina Siegesmund, BS, 1 Norbert Bru“ ggemann, MD,1 Alexander Schmidt, MD, 1 Meike Kasten, MD,1,2 Irene Pichler, PhD,3 Hiltrud Muhle, MD,4 Ebba Lohmann, MD,5 Thora Lohnau, BS, 1 Eberhard Schwinger, MD,6 Johann Hagenah, MD,1 Ulrich Stephani, MD,4 Peter P. Pramstaller, MD,3 Christine Klein, MD,1* and Katja Lohmann, PhD2012

36. Family-Based and Population-Based Association Studies Validate PTPRD as a Risk Factor for Restless Legs Syndrome. Published in final edited form as: Mov Disord. 2011 February 15; 26(3): 516-519. doi:10.1002/mds.23459.

37. Gamma-aminobutyric acid (GABA) receptors genes polymorphisms and risk for restless legs syndrome Article Published: 03 May 2018 Félix Javier Jiménez-Jiménez, Gara Esguevillas, Hortensia Alonso-Navarro, Martín Zurdo, Laura Turpín-Fenoll, Jorge Millán-Pascual, Teresa Adeva-Bartolomé, Esther Cubo, Francisco Navacerrada, Gemma Amo, Ana Rojo-Sebastián, Lluisa Rubio, Mónica DíezFairén, Pau Pastor, Marisol Calleja, José Francisco Plaza-Nieto, Belén Pilo-de-la-Fuente, Margarita Arroyo-Solera, Esteban García-Albea, José A. G. Agúndez \& Elena García-Martín The Pharmacogenomics Journal volume 18, pages565-577(2018)

38. Generation of a recombinant Newcastle disease virus expressing two foreign genes for use as a multivalent vaccine and gene therapy vector, Haixia Hu „, Jason P. Roth ,Qingzhong Yu, 30 June 2018

39. Genetic analysis of age at onset variation in spinocerebellar ataxia type 2, K.P. Figueroa, Hilary Coon, Nieves Santos, Luis Velazquez, Luis Almaguer Mederos, Stefan M. Pulst, May 15, 2017

40. Genetic and epidemiological characterization of restless legs syndrome in Québec Fulya Akçimen1,2, Jay P. Ross1,2, Faezeh Sarayloo1,2, Calwing Liao1,2,,Rachel De Barros Oliveira2, Jennifer A. Ruskey2,3,Cynthia V. Bourassa2,3, Patrick A. Dion2,3, Lan Xiong2,3 , Ziv Gan-Or1,2,3,Guy A. Rouleau1,2,3, Advance Access Publication Date: 30 October 2019 Original Article 
41. Genetic aspects of restless legs syndrome V Dhawan, M Ali, K R Chaudhuri. Postgrad Med J 2006;82:626-629. doi: 10.1136/pgmj.2006.045690

42. Genetic aspects of restless legs syndrome. V Dhawan, M Ali, K R Chaudhuri. Postgrad Med J 2006;82:626-629.oi:1136/pgmj.2006.045690 Iron and restless legs syndrome: treatment, genetics and athophysiology James R. Connor a, *, Stephanie M. Patton a, Konrad Oexle b, 1, Richard P. Allen c a Department of Neurosurgery, Penn State Hershey Medical Center, Hershey, PA, USA b Institut für Humangenetik, Technische Universit€at, Munich, Germany. Sleep Medicine 31 (2017).

43. Genetic association studies of neurotensin gene and restless legs syndrome in French Canadians. Lan Xiong a, Anastasia Levchenko a, Jacques Montplaisir b, Jean-Baptiste Rivie`re a, Pascale Thibodeau a, Judith St-Onge a, Claudia Gaspar a, Alex Desautels a,b, Paul Lespe'rance c, Sylvain Chouinard c, Gustavo Turecki d, Guy A. Rouleau.2008

44. Genetic markers of Restless Legs Syndrome in Parkinson Disease Ziv Gan-Or, MD, PhD1,2, Roy N. Alcalay, MD, MSc3, Anat Bar-Shira, PhD4, Claire S. Leblond, PhD1,2,

45. Genetics of restless legs syndrome (RLS): State-of-the-art and future directions Juliane Winkelmann MD Oli Polo MD Federica Provini MD Sonja Nevsimalova MD David Kemlink MD, PhD Karel Sonka MD Birgit Högl MD Werner Poewe MD Karin Stiasny-Kolster MD Wolfgang Oertel MD Al de Weerd MD Luigi Ferini Strambi MD Marco Zucconi MD Peter P. Pramstaller MD Isabelle Arnulf MD Claudia Trenkwalder MD Christine Klein MD Georgios M. Hadjigeorgiou MD Svenja Happe MD David Rye MD, PhD Pasquale Montagna MD-2007

46. Hasheminasab Zaware R, Mahmoodi Meymand M H, Rezaeian M, Mohammadi Kamalabadi N, Mostafavi S-A, Abdolkarimi Dawarani M A, Jome Yazdian R and Bidaki R (2016)

47. Hyperdopaminergism in lenticulostriate stroke-related restless legs syndrome: an imaging study, Elisabeth Ruppert, Marc Bataillard, Izzie Jacques Namer, Laurent Tatu,

48. Identification of a major susceptibility locus for restless legs syndrome on chromosome 12q, Desautels A1, Turecki G, Montplaisir J, Sequeira A, Verner A, Rouleau GA. 2001

49. Identification of a Major Susceptibility Locus for Restless Legs Syndrome on Chromosome 12q, Author links open overlay ,nelAlex Desautels 12 Gustavo Turecki 2 Jacques Montplaisir1 Adolfo Sequeira 2 Andrei Verner 3 Guy A. Rouleau, 2001

50. Identification of Restless Legs Syndrome Genes by Mutational Load Analysis, Erik Tilch PhD Barbara Schormair PhD Chen Zhao PhD Aaro V. Salminen PhD Ana Antic Nikolic MD Evi Holzknecht MD Birgit Högl MD Werner Poewe MD Cornelius G. Bachmann MD, 01 December 2019

51. Identifying risk factors for restless leg syndrome Dr. Sanjay Pandey

52. Insomnia and Restless Leg Syndrome in Patients Undergoing Chronic Hemodialysis in Rafsanjan Ali Ibn Abitaleb Hospital.

53. Juliane Winkelmann, MD,Helmholz Center Munich, National Research Center,for Environment and Health, Munich Institute of Human,Genetics, Ingolstaedter Landstrasse 1,D-85764 MunichNeuherberg, Germany.Current Neurology and Neuroscience Reports 2008, 8:211-216 
54. Lack of Association between Genetic Risk Loci for Restless Legs Syndrome and Multimorbidity.András Szentkirályi, MD, PhD1 Henry Völzke, MD2,3; Wolfgang Hoffmann, MD, MPH2,4; Julianne Winkelmann, MD5,6,7,8; Klaus Berger, MD, MPH, MSc1(2016)

55. MEIS1 and Restless Legs Syndrome: A Comprehensive Review. Faezeh Sarayloo, Patrick A. Dionand Guy A. Rouleau, 28 August 2019

56. MEIS1 variant as a determinant of autonomic imbalance in Restless Legs Syndrome, Jérôme Thireau, Charlotte Farah, Nicolas Molinari, Fabrice Bouilloux, Lucas Torreilles, Juliane Winkelmann, Sabine Scholz, Sylvain Richard, Yves Dauvilliers \& Frédéric Marmigère (2017)

57. Meis1: effects on motor phenotypes and the sensorimotor system in mice, Aaro V. Salminen, Lillian Garrett, Barbara Schormair, Jan Rozman, Florian Giesert, Kristina M. Niedermeier, Lore Becker, Birgit Rathkolb, Ildikó Rácz, German Mouse Clinic Consortium, Martin Klingenspor, Thomas Klopstock, Eckhard Wolf, Andreas Zimmer, Valérie Gailus-Durner, Miguel Torres, Helmut Fuchs, Martin Hrabě de Angelis, Wolfgang Wurst, Sabine M. Hölter, Juliane Winkelmann,Disease Models \& Mechanisms 2017

58. Moeller5,Lilian Lin1,2, Michael Mull6, Martin Ha“ usler7, Jo“ rg B. Schulz1,8, Joachim Weis2 \& Kristl G. Claeys1,2,9Deutsche Gesellschaft f€ur Muskelkranke (DGM) (Grant/Award Number: N/A).Received: 17 November 2015; Revised: 25 January 2016; Accepted: 2 February 2016

59. Molecular Genetic Studies of DMT1 on 12q in French-Canadian Restless Legs Syndrome Patients and Families. Lan Xiong,1 Patrick Dion,1 Jacques Montplaisir,2 Anastasia Levchenko,1 Pascale Thibodeau,1 Liliane Karemera,1 Jean-Baptiste Rivie` re,1 Judith StOnge,1 Claudia Gaspar,1 MariePierre Dube' ,3 Alex Desautels,1,2 Gustavo Turecki,4 and Guy A. Rouleau1*.American Journal of Medical Genetics Part B (Neuropsychiatric Genetics) 144B:911-917 (2007)

60. Movement Disorder Society Restless Legs Syndrome, Rapid Eye Movement Sleep Behavior Disorder, and Hypersomnia in Patients with Two Parkin Mutations. Nade ge Limousin, MD,1 Eric Konofal, MD, $\mathrm{PhD}, 1$ Elias Karroum, MD,1 Ebba Lohmann, MD,2 loannis Theodorou, MD, PhD,3 Alexandra Du“rr, MD, $\mathrm{PhD}, 2,4$ and Isabelle Arnulf, MD, PhD1*.2009

61. New concepts in the management of restless legs syndrome, Diego Garcia-Borreguero, neurologist and director, Irene Cano-Pumarega, head of pulmonology, (Published 27 February 2017)

62. Next-generation DNA sequencing identifies novel gene variants and pathways involved in specific language impairment, Xiaowei Sylvia Chen, Rose H. Reader, Alexander Hoischen, Joris A. Veltman, Nuala H. Simpson, Clyde Francks, Dianne F. Newbury \& Simon E. Fisher, (2017)

63. Non-manifesting Refsum heterozygotes carrying the c.135-2A>G PAHX gene transition Josef Finsterer • Günther Regelsberger • Till Voigtländer. Neurol Sci (2008) 29:173-175 DOI 10.1007/s10072-008-0931-4

64. Non-manifesting Refsum heterozygotes carrying the c.135-2A>G PAHX gene transitionJosef Finsterer - Günther Regelsberger • Till Voigtländer.2008

65. Not only limbs in atypical restless legs syndrome, Turrini A, Raggi A, Calandra-Buonaura G, Martinelli P, Ferri R, Provini F,Not Only Limbs in Atypical Restless Legs Syndrome, Sleep Medicine Reviews 
(2017),

66. Obesity: a possible risk factor for restless legs syndrome Demet YildizD, Nilufer Buyukkoyuncu,Ahmet kasim kilic,senor cander,Abdulmekit ylzdiz,Meral seferoglu,sevda erer uzbek, Received 16 May 2016, Accepted 27 Aug 2017, Published online: 25 Sep 2017

67. Opioids in the treatment of restless legs. syndrome: pharmacological and clinical aspects Stefano de Biase, Giovanni Merlino, Mariarosaria Valente \& Gian Luigi Gigli. tefano de Biase, Giovanni Merlino, Mariarosaria Valente \& Gian Luigi Gigli (2016)

68. Outcomes of long-term iron supplementation in pediatric restless legs syndrome/periodic limb movement disorder (RLS/PLMD), Thomas J. Dye a, b, ${ }^{*}$, Sejal V. Jain a, b, Narong imakajornboon,2017

69. Parkin Gene Modifies the Effect of RLS4 on the Age at Onset of Restless Legs Syndrome (RLS). Institute of Genetic Medicine, European Academy Bozen/Bolzano (EURAC), Bolzano, Italy; Affiliated Institute of the University of L€ubeck, Germany 2Department of Neurology, University of L€ubeck, Germany 3Department of Neurology, General Central Hospital, Bolzano, Italy Received 28 November 2008; Accepted 4 May 2009 Prevalence of Restless Legs Syndrome and Sleep Quality in Carriers of the Fragile X Premutation. Published in final edited form as: Clin Genet. 2014 August ; 86(2): 181184.

70. Periodic limb movements and restless legs syndrome in children with a history of prematurity, Christopher M. Cielo, Lourdes M. DelRosso, Ignacio E. Tapia, Sarah,N. Biggs, Gillian M. Nixon, Lisa J. Meltzer, Joel Traylor, Ji Young Kim,Carole L. Marcus, Caffeine for Apnea of Prematurity - Sleep Study Group,3/12/2016

71. Pharmacological Management of Restless Legs Syndrome and Periodic Limb Movement Disorder in Children, Geoffrey RulongThomas DyeNarong Simakajornboon, 22 August 2017

72. Prevalence of Restless Legs Syndrome and Sleep Quality in Carriers of the Fragile X Premutation Scott M Summers, MD, PhD1,2, Jennifer Cogswell, BA1,3, John E Goodrich, BA1,4, Yi Mu, MS5, Danh V. Nguyen, PhD6, Steven D. Brass, MD7, and Randi J. Hagerman, MD1,31Medical Clin Genet. 2014 August; 86(2): 181-184.

73. Prevalence of restless legs syndrome during detoxification from alcohol and opioids Author links open overlay panelSusan E.MackieM.D.abdR. Kathryn McHugh Ph.D. bcKatherineMcDermottB.A.cMargaret L.GriffinPh.D.bcJohn W.WinkelmanM.D., Ph.D.bd1Roger D.WeissM.D.bc1, Available online 6 October 2016.

74. Prevalence of restless legs syndrome during pregnancy: A systematic review and metaanalysisGiovanni Rizzo, Xu Li, Sebastiano Galantucci, Massimo Filippi, Yong Won Cho,OCTOBER2017

75. Prospective study of obesity, hypertension, high cholesterol and risk of Restless Legs Syndrome .Katerina De Vito1, Yanping Li2, Salma Batool-Anwar3, Yi Ning, MD4, Jiali Han2, and Xiang Gao2,5,61Department. Mov Disord. 2014 July ; 29(8): 
76. Relationship between the quality of sleep and restless legs syndrome among, Alidosti M, Hemate Z, Reisi M.. J Kashan Univ Med Sci (Feyz) 2013; 17(1): (Persian).

77. Restless Leg Syndrome/Willis-Ekbom Disease Pathophysiology .Richard P. Allen, PhD Department of Neurology, Johns Hopkins University, Asthma \& Allergy Building, 1B76b, 5501 Hopkins Bayview Boulevard, Baltimore, MD 21224, USA. Sleep Med Clin. 2015 September ; 10(3): 207-214. doi:10.1016/j.jsmc.2015.05.022.

78. Restless Leg Syndrome: Role of Iron and Vitamin D Deficiencies Department of Neurology, Near East University School of Medicine, Nicosia, Cite Cyprus this article as: Diker S. Restless Leg Syndrome: Role of Iron and Vitamin D Deficiencies. Cyprus J Med Sci 2018; 3: 114-6.

79. Restless Legs Syndrome and Sleep-Related Movement Disorders, Lynn Marie Trotti, MD, MSc,2017

80. Restless legs syndrome in patients with multiple sclerosis: restlesslegs syndrome in multiple sclerosis: evaluation of risk factors and clinical impact.2017

81. Restless legs syndrome in type 2 diabetes mellitus University of Health Sciences, Dr. Lütfi Kırdar Kartal Training and Research Hospital, Diabetes Center, Department of Internal Medicine, Istanbul, Turkey,University of Health Sciences, Dr. Lütfı Kırdar Kartal Training and Research Hospital, Department of Neurology, Istanbul, Turkey,Istanbul Medeniyet University, Department of Public Health, Istanbul, Turkey,2019

82. Restless legs syndrome Lisa Klingelhoefer, research and clinical fellow,A Kalyan Bhattacharya, professor,B and Heinz Reichmann, professorC, 2016 Aug

83. Restless legs syndrome. Lisa Klingelhoefer, A Kalyan BhattacharyaB and Heinz Reichmann CClinical Medicine 2016 Vol 16, No 4: 379-82

84. Restless Legs Syndrome: clinical features, diagnosis and a practical approach to management Subhashie Wijemanne1, William Ondo2, http://dx.doi.org/ 10.1136/ practneurol-2017-

85. Restless Legs Syndrome: Current Concepts about Disease Pathophysiology. Tremor Other Hyperkinet Mov. 2016

86. Restless legs syndrome: differential diagnosis and management with rotigotine, Giovanni Merlino1,3 Anna Serafini1 Francesca Robiony2 Mariarosaria Valente1,3 Gian Luigi Gigli1,2009

87. Risk of Cardiovascular Disease Associated with a Restless Legs Syndrome Diagnosis in a Retrospective Cohort Study from Kaiser Permanente Northern California Stephen K. Van Den Eeden, PhD1; Kathleen B. Albers, MPH1; Julie E. Davidson, PhD2; Clete A. Kushida, MD3; Amethyst D. Leimpeter, MS1; Lorene M. Nelson, PhD4; Rita Popat, PhD4; Caroline M. Tanner, MD, PhD5; Kristen Bibeau, PhD2; Charles P. Quesenberry, PhD1. SLEEP 2015;38(7):1009-.5101

88. Ron B. Postuma, MD, MSc5, Shay Ben-Shachar, MD4, Cheryl Waters, MD3, Amelie Johnson, MSc6, Oren Levy, MD, PhD3, Anat Mirelman, PhD7, Mali Gana-Weisz, PhD4, Nicolas Dupre, MD, MSc8, Jaques Montplaisir, MD, PhD9,10, Nir Giladi, MD7,11, Stanley Fahn, MD3, Lan Xiong, MD, PhD6,10,12, Patrick A. Dion, PhD1,12, Avi Orr-Urtreger, MD, PhD4,11, and Guy A. Rouleau, MD, PhD1,121Montreal. Parkinsonism Relat Disord. 2015 June ; 21(6): 582-585. doi:10.1016/j.parkreldis.2015.03.010. 
89. Segregation Analysis of Restless Legs Syndrome: Possible Evidence for a Major Gene in a Family Study Using Blinded Diagnoses, Mathias R.A.a · Hening W.b · Washburn M.c · Allen R.P.c · Lesage S.c - Wilson A.F.a · Earley C.J.cAuthor affiliations, (2006)

90. Targeting the dopamine D3 receptor: an overview of drug design strategies

91. Terry Kit Selfe, Sijin Wen, Karen Sherman, Maryanna Klatt \& Kim E. Innes ,Trials volume 20, Article number: 134 (2019)

92. The Association Between Subclinical Hypothyroidism and Sleep Quality: A Population-Based Study Linlin Song,1 Jianyong Lei,1 Ke Jiang,1 Yali Lei,2 Yuting Tang,2 Jingqiang Zhu,1 Zhihui Li,1 and Huairong Tang2. Risk Manag Healthc Policy. 2019; 12: 369-374.Published online 2019 Dec 19.

93. The Association Of Meis1 Gene In Restless Leg Syndrome And Rls Related Phenotypes But Not With Chronic Insomnia Disorder, M El Gewely; Melanie, W; Lan, X; Sophie, Y; Rouleau, G; et al. (Apr 2018)

94. The BTBD9 gene polymorphisms in Polish patients with Gilles de la Tourette syndrome. Janik P1, Berdyński M, Safranow K, Zekanowski C. 2014

95. The Comorbidity of Migraine and Restless Legs Syndrome, Semiha Kurt, September 2019

96. The Relationship Between RestlessLegs Syndrome and Anxiety, Depression, and Quality of Life Şenay Aydın,1 Cengiz Özdemir2.2019

97. The solute carrier family 1 (glial high affinity glutamate transporter), member 2 gene, SLC1A2, rs3794087 variant and assessment risk for restless legs syndrome, Author links open overlay panelFélix JavierJiménez-JiménezabHortensiaAlonsoavarroabCarmenMartínezcMartínZurdodLauraTurpín-FenolleJorgeMillán-PascualeTeresaAdevaBartoloméfEstherCubogFranciscoNavacerradaaAnaRojoSebastiánbLluisaRubiobMarisolCallejaaJosé FranciscoPlaza-NietoaBelénPilo-de-laFuenteaMargaritaArroyo-SoleraaElenaGarcía-MartínhJosé A.G.Agúndezi, (2013)

98. Thr105lle (rs11558538) polymorphism in the histamine-1-methyl-transferase (HNMT) gene and risk for restless legs syndrome, Jiménez-Jiménez FJ1,2, García-Martín E3, Alonso-Navarro H4,5, Martínez C6, Zurdo M7, Turpín-Fenoll L8, Millán-Pascual J8, Adeva-Bartolomé T9, Cubo E10, Navacerrada F4, Rojo-Sebastián A5, Rubio L5, Ortega-Cubero S11,12,13, Pastor P11,12,13,14, Calleja M4, Plaza-Nieto JF4, Pilo-de-la-Fuente B4, Arroyo-Solera M4, García-Albea E5, Agúndez JA3. (2017)

99. TOX3 Variants Are Involved in Restless Legs Syndrome and Parkinson's Disease with Opposite Effects, Sadaf MohtashamiQin HeJennifer A. RuskeySirui ZhouPatrick A. DionRichard P. AllenChristopher J. EarleyEdward A. FonLan XiongNicolas DupreYves DauvilliersGuy A. RouleauZiv Gan- 05 February 2018

100. Underestimated associated features in CMT neuropathies clinical indicators for the causative gene? Friederike Werheid1,2, Hamid Azzedine2, Eva Zwerenz1,2, Ahmet Bozkurt3,4, Marcus J.

101. y Emilia Sforza,Frédéric Roche andVincent Pichot *,Published: 4 October 2019 Alibabaei, Z. (2018). Restless leg syndrome in Iranian family.

\section{Tables}


Table 1- The frequency distribution of genes: TANC1,MEIS1 AND ATXN7

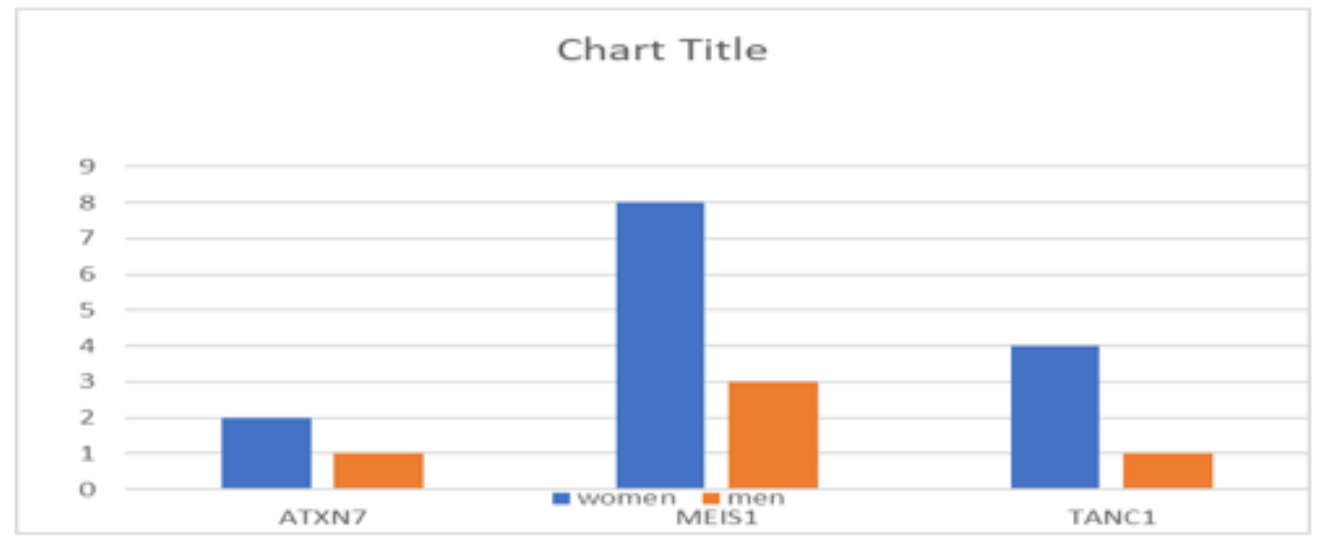

Table 2.Table of age frequency in the subjects

\begin{tabular}{cr|r|r|r|r}
\hline Valid & & & & \\
\cline { 2 - 6 } & $10-20$ & 6 & 12.8 & 12.8 & 12.8 \\
\hline $20-30$ & 2 & 4.3 & 4.3 & 17.0 \\
\hline $30-40$ & 15 & 31.9 & 31.9 & 48.9 \\
\hline $40-50$ & 14 & 29.8 & 29.8 & 78.7 \\
\hline $50-60$ & 6 & 12.8 & 12.8 & 91.5 \\
\hline $60-70$ & 4 & 8.5 & 8.5 & 100.0 \\
\hline Total & 47 & 100.0 & 100.0 & \\
\hline
\end{tabular}

Table3-Comparison of studied genes 


\begin{tabular}{|c|c|c|c|c|c|}
\hline Gene & Variant & DANN & chassification & Mutation tester & Publication ferequency \\
\hline \multirow{7}{*}{ A TXN7 } & G183A & Pathogenic & Uncertain Significance & disease causing & $f=0.00000634$ \\
\hline & G191A & Damaging & Likely Benign & Polymorphism & $f=0.0000057$ \\
\hline & $196 \mathrm{G}>\mathrm{T}$ & Tolerated & Likely Benign & Polymorphism & $44.58 \%$ \\
\hline & $215 \mathrm{C}>\mathrm{T}$ & Pathogenic & Unknown & Polymorphism & $f=0.00000997$ \\
\hline & $240 \mathrm{G}>\mathrm{A}$ & Pathogenic & Uncertain Significance & disease causing & $f=0.00000421$ \\
\hline & $242 \mathrm{G}>\mathrm{A}$ & Disease causing & Benign & disease causing & $f=0.000021$ \\
\hline & $211 \mathrm{~T}>\mathrm{C}$ & Pathogenic & Likely Benign & Polymorphism & $f=0.00001$ \\
\hline TANC1 & $183 \mathrm{G}>\mathrm{A}$ & Disease causing & Uncertain Significance & disease causing & $f=0.000016$ \\
\hline \multirow{12}{*}{ MEIS1 } & $18 \mathrm{C}>\mathrm{A}$ & disease causing & Uncertain Significance & disease causing & $f=0.00000403$ \\
\hline & $33 \mathrm{C}>\mathrm{T}$ & Pathogenic & Uncertain Significance & disease causing & $f=0.0000121$ \\
\hline & $45 \mathrm{~T}>\mathrm{C}$ & Pathogenic & Uncertain Significance & disease causing & $f=0.0000121$ \\
\hline & $56 \mathrm{~T}>\mathrm{G}$ & Pathogenic & Uncertain Significance & disease causing & $f=0.00000804$ \\
\hline & $69 \mathrm{G}>A$ & Damaging & Uncertain Significance & disease causing & $f=0.00000803$ \\
\hline & $78 \mathrm{C}>\mathrm{A}$ & Damaging & Likely Benign & disease causing & $f=0.000189$ \\
\hline & $114 \mathrm{C}>\mathrm{T}$ & Pathogenic & Uncertain Significance & disease causing & $f=0.00000402$ \\
\hline & $134 \mathrm{~T}>\mathrm{G}$ & Damaging & Uncertain Significance & disease causing & $f=0.00000404$ \\
\hline & $141 \mathrm{G}>\mathrm{C}$ & Pathogenic & Uncertain Significance & disease causing & $f=0.0000081$ \\
\hline & $149 A>G$ & Disease causing & Uncertain Significance & disease causing & $f=0.00000407$ \\
\hline & $188 \mathrm{~T}>\mathrm{A}$ & Disease causing & Uncertain Significance & disease causing & $f=0.00000436$ \\
\hline & $207 \mathrm{C}>\mathrm{T}$ & Pathogenic & Likely Benign & disease causing & $f=0.000335$ \\
\hline
\end{tabular}

Table4.Table of frequency of pathogens in the subjects

\begin{tabular}{|lllll|}
\hline & $\begin{array}{l}\text { Frequency } \\
\text { percent }\end{array}$ & percent & valid & Cumulative percent \\
& & & & \\
& 21 & 44.7 & 44.7 & 44.7 \\
\hline patient & 11 & 23.4 & 23.4 & 68.1 \\
\hline Healthy & 15 & 31.9 & 31.9 & 100.0 \\
\hline Carrier of pathogenic genes & 47 & 100.0 & 100.0 & \\
\hline total & & & & \\
\hline
\end{tabular}

\section{Figures}




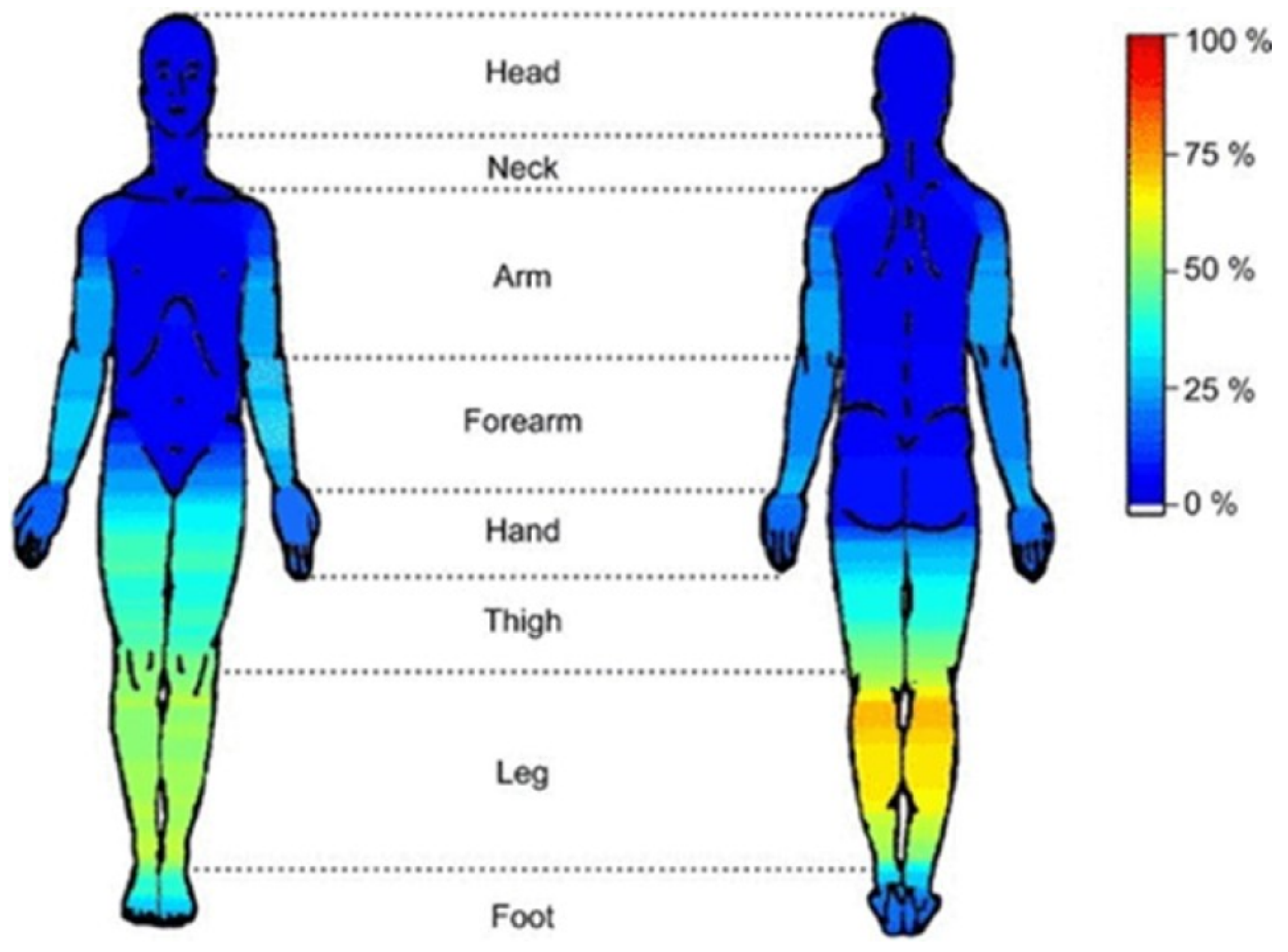

Figure 1

Diagrams of parts of the body that show signs of restless legs syndrome 